\title{
Inverting the Reflectance Map with Binary Search
}

\author{
François Faure \\ faure@imag.fr \\ iMAGIS/IMAG ${ }^{\star}$, BP53 38041 Grenoble cedex 9 \\ tel +3376635794 , fax +3376446675
}

\begin{abstract}
Stereophotometric computer vision is based on the inversion of an equation system which represents the physical reflection phenomenon under different lighting conditions. In noisy conditions, the system has generally no solution, and the typical way to solve it is to find the solution which minimizes some error function. This paper proposes a new approach to deal with this system, using the so-called Geometrical Compatibility Function. The problem is turned into a root-finding in a one-dimensional space and the robustness is largely improved with respect to wrong reflectance model coefficients.
\end{abstract}

\section{Introduction}

\subsection{BRDF and the surface model}

Stereophotometric methods for computer vision, first introduced by Woodham [7], aim to locally compute from different images the tangent plane at each point of a surface, in order to deduce a surface model. They are based on the knowledge of the Bidirectionnal Reflection Distribution Function (BRDF), which represents the scattering of reflected light through the directions of the local exterior halfspace (fig. 1).

Most real-world materials do not have the Lambertian property which leads to linear equations. There are typically two kinds of non-Lambertian phenomenons: specularities and specular diffuse reflection. Specularities (mirror-like reflections) were much studied in order to avoid their influence on the computation, or to use them for geometrical deductions $[1,2,3]$. The approach is to treat them apart from diffuse reflection. The specular diffuse reflection is difficult to deal with, since it needs knowledge of the BRDF and involves more complex and unstable equations. It was shown [4] that for a large class of BRDFs, three equations ( thus three images ) are needed to obtain a unique solution. The problem is that the BRDF is usually unknown, thus approximations lead to errors in the reconstruction of the normals.

\footnotetext{
* iMAGIS is a joint project CNRS,IMAG, INPG, INRIA, UJF
} 


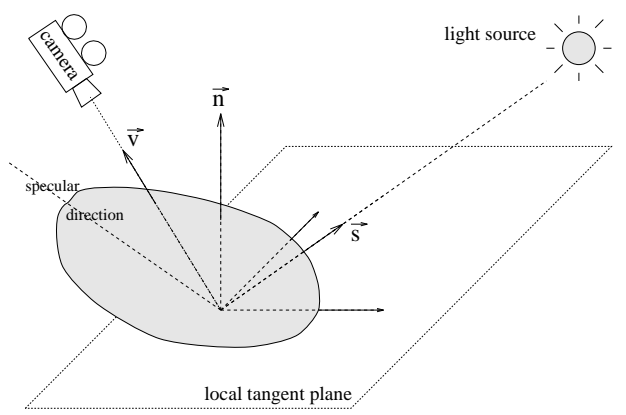

Fig. 1. The grey 3d-surface represents the values of a plausible non-Lambertian $B R D F(\boldsymbol{n}, \boldsymbol{s}, \boldsymbol{v})$ for different viewing directions

\subsection{Computing the normals}

The input data is composed of several images created under different known lighting conditions. From a physical point of view $[6,5]$, radiance $L_{r}$ cast through direction $\boldsymbol{v}$ is quantified by the expression:

$$
L_{r}(\boldsymbol{n}, \boldsymbol{v})=\int_{\Omega} L(\boldsymbol{s}) \boldsymbol{n} . \boldsymbol{s} B R D F(\boldsymbol{n}, \boldsymbol{s}, \boldsymbol{v}) d \omega_{i}
$$

where $L(\boldsymbol{s})$ is the irradiance from direction $s$, expressed in $W \cdot m^{-2} . s t^{-1}$, and $\Omega$ the exterior half-space. When the $B R D F$ is known, and with three available images, equation 1 and numerical data allow us to build equation systems, the solution of which provide the local normal coordinates on a regular grid $\boldsymbol{n}(i, j)$ which corresponds to the pixels grid.

\section{A new method for computing the normals}

\subsection{Creating the equation system}

In order to simplify equations, the light source is typically assumed to be a single point at infinity, of known power, and we will only deal with diffuse reflection, without interreflections. The radiance equation 1 becomes $L_{r}(\boldsymbol{s}, \boldsymbol{n}, \boldsymbol{v})=$ $L(\boldsymbol{s}) \boldsymbol{n} . \boldsymbol{s} B R D F(\boldsymbol{n}, \boldsymbol{s}, \boldsymbol{v})$ with $L(\boldsymbol{s})$ expressed in $W \cdot m^{-2}$. In the case of a nonLambertian reflection $[8,9,10]$, the system:

$$
\left\{\begin{array}{l}
L_{r}\left(\boldsymbol{s}_{1}, \boldsymbol{n}, \boldsymbol{v}\right)=L_{1} \\
L_{r}\left(\boldsymbol{s}_{2}, \boldsymbol{n}, \boldsymbol{v}\right)=L_{2} \\
L_{r}\left(\boldsymbol{s}_{3}, \boldsymbol{n}, \boldsymbol{v}\right)=L_{3}
\end{array}\right.
$$

is not linear, and has no root in case of noisy data or inappropriate BRDF. With a two-equation system, there are two solutions in the general case. The classical approach is to minimize some cost function like the quadratic difference between estimated and measured radiances. The dimensions of the search space may be two or three, depending on how you parametrize the normal. 


\subsection{Root-Finding Resolution}

Principle. Most BRDFs used for reconstruction are functions of $\boldsymbol{n . v}$ and $\boldsymbol{n . s}$, like those of Blinn, or Torrance-Sparrow. We will use one suggested by Lewis [5], for its physically plausible analytical form, which leads to the radiance relation

$$
L(\boldsymbol{n}, \boldsymbol{s}, \boldsymbol{v})=L(\boldsymbol{s}) \boldsymbol{n} . \boldsymbol{s}\left(K_{d}+K_{s}\left(\frac{\boldsymbol{n} \cdot \boldsymbol{s}+\boldsymbol{n} \cdot \boldsymbol{v}}{\|\boldsymbol{s}+\boldsymbol{v}\|}\right)^{n}\right)
$$

where $K_{d}, K_{s}$ and $n$ are independent real coefficients. The first one corresponds to the Lambertian part of the reflection, the others define what is usually called the forescatter lobe.

Notice that this radiance function is monotonically increasing with respect to $\boldsymbol{n . s}$ and $\boldsymbol{n . v}$. This means that, for measured values $R$ and $L(\boldsymbol{s})$, given a certain $\boldsymbol{n . v}$, you can find the corresponding $\boldsymbol{n . s}=n s(\boldsymbol{n} . \boldsymbol{v})$ with a binary search. The problem is to know the value of $\boldsymbol{n . v}$. For three images taken with a fixed camera and different known lighting directions, it is easy to compute the $a_{i}$ coefficients such that $\boldsymbol{v}=a_{1} \boldsymbol{s}_{1}+a_{2} \boldsymbol{s}_{2}+a_{3} \boldsymbol{s}_{3}$. The linearity of the dot product leads to $\boldsymbol{n . v}=a_{1} \boldsymbol{n} . \boldsymbol{s}_{1}+a_{2} \boldsymbol{n} . \boldsymbol{s}_{2}+a_{3} \boldsymbol{n} . \boldsymbol{s}_{3}$. Replacing the $n s_{i}(\boldsymbol{n} . \boldsymbol{v})$ functions in the above relation creates a function that we will call the Geometrical Compatibility Function (GCF), and the desired value must be a root of this function (fig.2): $a_{1} n s_{1}(\boldsymbol{n} . \boldsymbol{v})+a_{2} n s_{2}(\boldsymbol{n} . \boldsymbol{v})+a_{3} n s_{3}(\boldsymbol{n} . \boldsymbol{v})-\boldsymbol{n . v}=0$

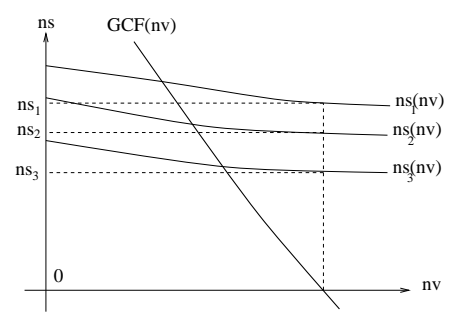

Fig. 2. The root provides the desired $n s_{i}$ values

Validity of the method. The necessary condition to create the Geometrical Compatibility Function is that the light directions must be linearly independent. A sufficient condition for the uniqueness of the root is that all the $a_{i}$ were positive, and the BRDF monotonic with respect to $\boldsymbol{n . v}$ and $\boldsymbol{n . s .}$. It means that for most reflection models, with the camera inside the $\left(s_{1}, s_{2}, s_{3}\right)$ trihedron, we can implement efficient binary search resolutions.

It might seem strange that the radiance equations system has no root whereas the above function does. The reason is that we did not enforce the $\|\boldsymbol{n}\|=1$ condition. This allows us to define a measure of quality defined as $e=\left(\boldsymbol{n}^{2}-1\right)^{2}$. This error will further be denoted as estimated error. The normal is then renormalized. Numerical experiments detailed further show that it is a reliable quality 
evaluator. Compared with a minimization, a root-finding has the advantage to provide an exact bracketing of the solution for any desired precision. We can also expect a quicker convergence.

Extension to different points of view This method is based on the fact that $\boldsymbol{n} \boldsymbol{v}$ is the same for each image when you change only the lighting source. For three images with the same source and different camera positions, the geometrical compatibility equation turns into $\boldsymbol{s}=a_{1} \boldsymbol{v}_{1}+a_{2} \boldsymbol{v}_{2}+a_{3} \boldsymbol{v}_{3}$ and the method can be adapted.

\section{Experimentation}

The minimization was implemented with a Powell's algorithm, whereas the rootfinding used a Newton-Raphson algorithm [11]. Numerous tries were made to compare the robustness of the two methods. A theoretical $64 \times 64$ surface was created, represented by points and normals. Three illuminations were simulated, the radiances for each pixel were computed with radiance equation 2 .

The important features are robustness with respect to false reflection coefficients $\left(K_{d}, K_{s}, n\right)$ and noisy data. The error function we use is the standard deviation between the initial and reconstructed normals. This value will be useful for two reasons: measuring the quality of the reconstruction, and if the estimated error is correctly related to the real error. It is actually important to know whether the estimated error is reliable or not, because if it is, we can expect to improve our reconstructions by minimizing the error with respect to the reflection coefficients.

The following figures show the influence of a wrong $K_{s}$ on the accuracy of reconstruction. The surface is Lambertian when $K_{s}=0$. The curves are very similar for $K_{d}$. For wrong $n$ values, both methods lead almost to the same result. The horizontal axis shows the different values $K_{s}$, whereas $K_{d}$ and $n$ are correct. The vertical axis shows the average deviation in degrees of the objective errors of the two methods, and the value of the estimated error. For clarity, the estimated error of the classical approach was not drawn.

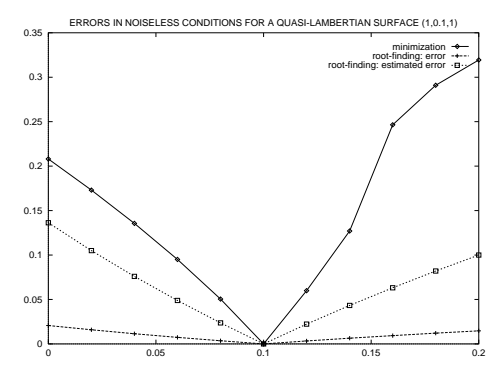

Fig. 3.

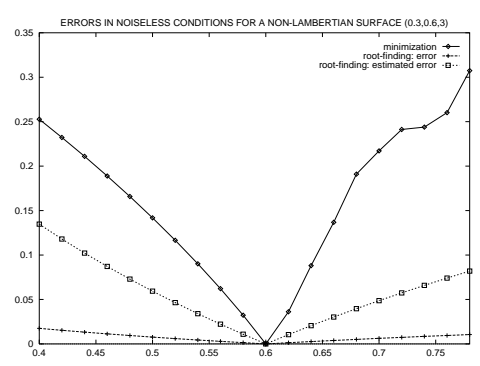

Fig. 4. 
The above figures show the different errors in noiseless conditions for a quasiLambertian surface (fig. 3) and for a non-Lambertian one(fig. 4). We can see that root-finding is much less sensitive to wrong coefficients. The estimated error can lead to the exact ones.

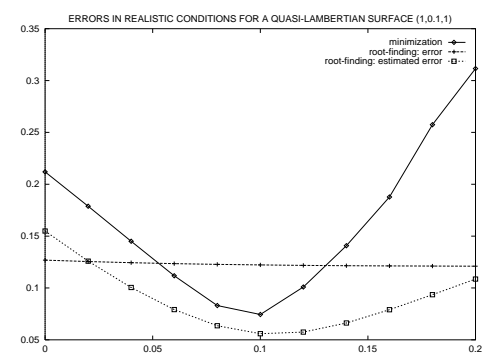

Fig. 5.

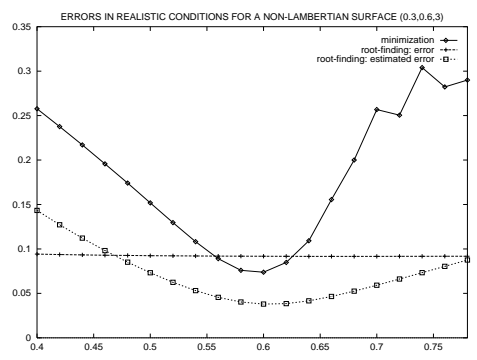

Fig. 6.

Figures 5 and 6 show the results with the same surfaces and a white, Gaussian noise with a standard deviation of two percent, which is a sensible value for modelizing real conditions. The new approach seems more sensitive to noisy data, since the error is bigger around the exact coefficients. However, wrong coefficients quickly compensate and reverse the difference. It means that in ordinary conditions we can expect a better reconstruction with unknown coefficients. Furthermore, the estimated error keeps a minimum around the exact coefficient, this will be helpful to improve it. Further tries showed that this minimum corresponds more precisely to the exact coefficients than the estimated error of the classical approach. Once we have the best coefficients, we can always improve the result by minimization.

The total time, including normal reconstruction, integrabilization, and integration is less than five minutes for a $512 \times 512$ surface on an standard SGI INDY workstation.

\section{Conclusion}

Considering the Geometrical Compatibility Equation allows us to turn the normal reconstruction into a root-finding problem. For a large class of reflectance models, the root is unique and can be found with binary search. This approach is continuously suitable from Lambertian to non-Lambertian surfaces. Different tries were made to compare the efficiency of this method with a classical approach under the same hypotheses. The root-finding seems more sensitive to noisy data but much less with respect to wrong reflectance coefficients. The provided estimated error is smooth and has one minimum around the exact coefficients. This new approach could be useful for non-Lambertian or quasi-Lambertian surfaces, whose reflectance function is almost always unknown. 


\section{References}

1. Katsushi Ikeuchi Determining surface orientations of specular surfaces by using the photometric stereo method IEEE PAMI-3, No 6, November 1991

2. S. W. Lee and R. Bajcsy Detection of Specularity Using Color and Multiple Views GRASP Laboratory, Philadelphia, PA 19082

3. G. J. Klinker, S. A. Shafer and T. Kanade The Measurement of Highlight in Color Images IJCV 2. 7-32, 1988

4. H. D. Tagare and R.J.P. de Figueiredo A Theory of Photometric Stereo for a Class of Diffuse Non-Lambertian Surfaces IEEE PAMI Vol 13, No 2, February 1991

5. R.R. Lewis Making shaders more physically plausible Fourth Eurographics Workshop on Rendering, Paris, 1993.

6. M. Oren and S.K. Nayar Seeing beyond Lambert's law eccv 1994

7. R.J.Woodham Photometric method for determining surface orientation from multiple images Optical Engineering, 19, 1980, p.139

8. J. F. Blinn Models of light reflection for computer synthesized pictures ACM Computer Graphics (SIGGRAPH 77), 19(10):542-547, 1977

9. K. Torrance and E. Sparrow Theory for off-specular reflection from rough surfaces Journal of the Optical Society of America, 57:1105-1114, September 1967

10. X.D.He, K.E. Torrance, F.X. Sillion,D.P. Greenberg A comprehensive physical model for light reflection Computer Graphics, Vol. 20, No 4, pp. 143-150, August 1986

11. Press, Teukolski, Vetterling and Flannery Numerical Recipes in C Cambridge University Press

This article was processed using the $\mathrm{IAT}_{\mathrm{E}} \mathrm{X}$ macro package with LLNCS style 